\title{
Psyschoonkologische Beratungsstellen der Deutschen Krebsgesellschaft e. V.
}

Die Landeskrebsgesellschaften der Deutschen Krebsgesellschaft haben ein Netz von psychosozialen Beratungsstellen in ganz Deutschland aufgebaut. Betroffene und ihre Angehörigen finden hier Informationen und praktische Hilfe - persönlich, telefonisch oder auch schriftlich.

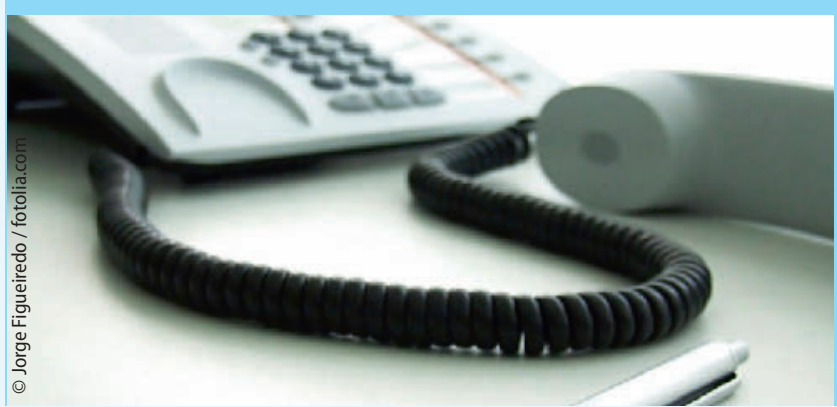

Eine Krebserkrankung wirft eine Fülle von Fragen auf. Die Mitarbeiter der Beratungsstellen sind dank ihrer fachlichen Kompetenz und ihrer Erfahrung in der Lage, Antworten auf Ihre Fragen zu finden. Sie helfen bei konkreten, aktuellen Lebensproblemen, die durch Erkrankungen entstehen oder verschärft werden:

- Umgang mit der Krankheit,

- Lebenshilfe bei Trauer und Verlust,

- Hilfe und Unterstützung bei Ängsten,

- Ernährung und Sport,

- Familien- und Paarkonflikte,

- Sexualität,

- Veränderung sozialer Kontakte,

Konflikte mit Ärzten,

- Schwierigkeiten im Klinikalltag

\section{$>$ Baden-Württemberg}

Krebsverband

Baden-Württemberg e.V.

Adalbert-Stifter-Straße 105

70437 Stuttgart

Tel.: 071184810770

info@krebsverband-bw.de

www.krebsverband-bw.de

D Bayern

Bayerische Krebsgesellschaft e. V.

Nymphenburger Straße $21 \mathrm{~A}$

80335 München

Tel.: 089 548840-0

Pressestelle@bayerischekrebsgesellschaft.de

www.bayerische-krebsgesellschaft.de

\section{$\nabla$ Berlin}

Berliner Krebsgesellschaft e. V.

Robert-Koch-Platz 7

10115 Berlin

Tel.: 0302832400

info@berliner-krebsgesellschaft.de

www.berliner-krebsgesellschaft.de

\section{Brandenburg}

Brandenburgische

Krebsgesellschaft e.V.

Charlottenstraße 57

14467 Potsdam

Tel.: 0331864806

mail@krebsgesellschaft-brandenburg.de

www.krebsgesellschaft-brandenburg.de
Bremen

Bremer Krebsgesellschaft e. V. Am Schwarzen Meer 101-105 28205 Bremen

Tel. 04214919222

bremerkrebsgesellschaft@t-online.de www.bremerkrebsgesellschaft.de

\section{\ Hamburg}

Hamburger Krebsgesellschaft e.V. Butenfeld 18

22529 Hamburg

Tel.: 0404604222

info@krebshamburg.de

www.krebshamburg.de

\section{> Hessen}

Hessische Krebsgesellschaft e. V. Heinrich-Heine-Straße 44 35039 Marburg

Tel.: 0642163324

oeffentlichkeitsarbeit@hessischekrebsgesellschaft.de

www.hessische-krebsgesellschaft.de

\section{\ Mecklenburg-Vorpommern}

DKG Landesverband

Mecklenburg-Vorpommern e. V.

Universitäts-Klinikum der

Ernst-Moritz-Arndt-Universität

Fr-Loeffler-Straße 23a

17475 Greifswald

Tel.: 01714014480

diemer-w@uni-greifswald.de

www.krebsgesellschaft-mv.de

\section{Diedersachsen}

Niedersächsische

Krebsgesellschaft e.V.

Königstraße 27

30175 Hannover

Tel.: 05113885262

service@nds-krebsgesellschaft.de

www.nds-krebsgesellschaft.de

D Nordrhein-Westfalen

Krebsgesellschaft Nordrhein-

Westfalen e. V.

Volmerswerther Straße 20

40221 Düsseldorf

Tel.: 021115760990

info@krebsgesellschaft-nrw.de

www.krebsgesellschaft-nrw.de

\section{Rheinland-Pfalz}

Krebsgesellschaft Rheinland-Pfalz

e. V.

Löhrstraße 119

56068 Koblenz

Tel.: 0261988650

kontakt@krebsgesellschaft-rlp.de

www.krebsgesellschaft-rlp.de

\section{\Saarland}

Saarländische

Krebsgesellschaft e.V.

Kuselerstraße 28

66564 Ottweiler

Tel.: 068588251

dr.lilo.ost@t-online.de

www.saarlaendische-krebsgesellschaft.de

\section{Dachsen}

Sächsische Krebsgesellschaft e. V. Haus der Vereine, Schlobigplatz 23 08056 Zwickau

Tel. 0375281403 info@skg-ev.de

www.saechsische-krebsgesellschaft-ev.de

\section{Sachsen-Anhalt}

Sachsen-Anhaltische Krebsgesellschaft e.V.

Paracelsusstraße 23

06114 Halle

Tel.: 03454788110

info@krebsgesellschaft-sachsen-anhalt.de www.krebsgesellschaft-sachsen-anhalt.de

\section{\Schleswig-Holstein}

Schleswig-Holsteinische Krebsgesellschaft e. V.

Holstenstraße 13-15

24103 Kiel

Tel.: 04318001080

info@krebsgesellschaft-sh.de www.krebsgesellschaft-sh.de

\section{$\nabla$ Thüringen}

Thüringische Krebsgesellschaft e. V. Felix-Auerbach-Straße 14 07747 Jena

Tel. 03641336986

info@thueringische-krebsgesellschaft.de www.thueringische-krebsgesellschaft.de 